\title{
Exome sequencing discloses KALRN homozygous variant as likely cause of intellectual disability and short stature in a consanguineous pedigree
}

\author{
Periklis Makrythanasis ${ }^{1,2}$, Michel Guipponi ${ }^{2}$, Federico A. Santoni ${ }^{1}$, Maha Zaki ${ }^{3}$, Mahmoud Y. Issa ${ }^{3}$, \\ Muhammad Ansar ${ }^{1}$, Hanan Hamamy ${ }^{1 *}$ and Stylianos E. Antonarakis ${ }^{1,2,4^{*}}$
}

\begin{abstract}
Background: The recent availability of whole-exome sequencing has opened new possibilities for the evaluation of individuals with genetically undiagnosed intellectual disability.

Results: We report two affected siblings, offspring of first-cousin parents, with intellectual disability, hypotonia, short stature, growth hormone deficiency, and delayed bone age. All members of the nuclear family were genotyped, and exome sequencing was performed in one of the affected individuals. We used an in-house algorithm (CATCH v1.1) that combines homozygosity mapping with exome sequencing results and provides a list of candidate variants. One identified novel homozygous missense variant in KALRN (NM_003947.4:c.3644C>A: p.(Thr1215Lys)) was predicted to be pathogenic by all pathogenicity prediction software used (SIFT, PolyPhen, Mutation Taster). KALRN encodes the protein kalirin, which is a GTP-exchange factor protein with a reported role in cytoskeletal remodeling and dendritic spine formation in neurons. It is known that mice with ablation of Kalrn exhibit age-dependent functional deficits and behavioral phenotypes.
\end{abstract}

Conclusion: Exome sequencing provided initial evidence linking KALRN to monogenic intellectual disability in man, and we propose that KALRN is the causative gene for the autosomal recessive phenotype in this family.

Keywords: Exome sequencing, Intellectual disability, Short stature, Consanguineous, KALRN

\section{Background}

Consanguinity is practiced in a large number of human populations with rates reaching $20-50 \%$ in several countries in North Africa and the Middle East [1, 2]. Offspring of consanguineous marriages are at a higher risk of having congenital anomalies caused by pathogenic variants in genes following autosomal recessive inheritance [3-5]. In a study in our laboratory, we employed whole-exome sequencing and genotype analysis to screen members of consanguineous families with likely recessive disorders. Our hypothesis was that because of the homozygosity of the causative defect, the

\footnotetext{
* Correspondence: hananhamamy@yahoo.com; Hanan.AlHamami@unige.ch; Stylianos.Antonarakis@unige.ch

${ }^{1}$ Department of Genetic Medicine and Development, University of Geneva, 1 Rue Michel-Servet, 1211 Geneva, Switzerland

Full list of author information is available at the end of the article
}

diagnostic strategy would be successful in identifying the molecular basis of the disorder in at least a proportion of the participating patients. Exome sequencing identified the causative variant in up to $34 \%$ of the progeny of consanguineous parents affected by undiagnosed autosomal recessive disorders sequenced in our laboratory [6], as well as a number of novel candidate genes for autosomal recessive disorders [7-9].

Early-onset intellectual disability originating before the age of 18 years with an IQ below 70 is estimated to affect $1-3 \%$ of western populations but could be more common in highly consanguineous populations [10] due to the role of autosomal recessive variants. Autosomal recessive intellectual disability (ARID) is extremely heterogeneous, and causative genes may reach thousands with the vast majority still unknown [10]. With the 
introduction of NGS technologies, new ID genes are being identified including over 300 genes for ARID [11]. Many of these were identified in studies on consanguineous families. Among 136 consanguineous Iranian families with various forms of ID, pathogenic variants were identified in 78 families including homozygous mutations in 23 genes previously implicated in ID and in 50 candidate genes for ARID [12]. Examples from recent studies on consanguineous families with ID revealing pathogenic variants include variants in TNIK gene [13], SLC6A17 gene [14], and c12orf4 gene [15].

To add to the repository of ARID candidate genes, we report two affected siblings, offspring of a consanguineous marriage, with intellectual disability (ID), hypotonia, short stature, growth hormone deficiency, and delayed bone age. Exome sequencing and homozygosity mapping identified only one strong candidate variant in KALRN (NM_003947.4:c.3644C>A: p.(Thr1215Lys)). Kalirin-7, a major isoform of kalirin, is known to regulate spine density in hippocampal and cortical neurons [16], growth hormone functional secretion [17], and bone homeostasis [18].

\section{Methods}

Genotyping, exome sequencing, and variant analysis were performed as previously described [6]. All the family members were genotyped with a dense genotype array in order to define runs of homozygosity $(\mathrm{ROH})$ in all the family members. DNA from the proband was used for exome sequencing, and after calling variants, the $\mathrm{ROH}$ were used in order to identify the variants that were in an $\mathrm{ROH}$ in the affected siblings, which was not shared by the unaffected sibling and the parents. In more detail, DNA samples from affected family members, their unaffected sibling, and their parents were genotyped using the HumanOmniExpress Bead Chip by Illumina Inc. ${ }^{\circ}$ (720K SNPs). ROH for every individual were identified using PLINK. We defined as $\mathrm{ROH}$ the regions with 50 consecutive SNPs irrespective of the total size of the genomic region, allowing for one mismatch. The $\mathrm{ROH}$ region was the one demarcated by the first heterozygous SNPs flanking each established homozygous region. The exome of one affected individual (IV:1) was captured using the SureSelect Human All Exonsv5 reagents by Agilent Inc. ${ }^{\circ}$. Sequencing was performed in an Illumina HiSeq 2000 sequencer. Results were analyzed with BWA [19], SAMtools [19], Pindel [20] and ANNOVAR [21], and the exonic variants in combination with the $\mathrm{ROH}$ were used by $\mathrm{CATCH}$ v1.1 [22] that provided the final list of variants

\section{Results \\ Clinical report}

Figure 1 shows the pedigree of the consanguineous family originating from Egypt. The parents of two affected offspring are paternal first cousins. The eldest affected is a girl (IV:1), first seen at the genetic clinic at the age of 13 years with intellectual disability, short stature, and delayed puberty. Her height was $128 \mathrm{~cm}$ (-5.1SD), weight was $25 \mathrm{~kg}(-2.3 \mathrm{SD})$, and head circumference $(\mathrm{HC})$

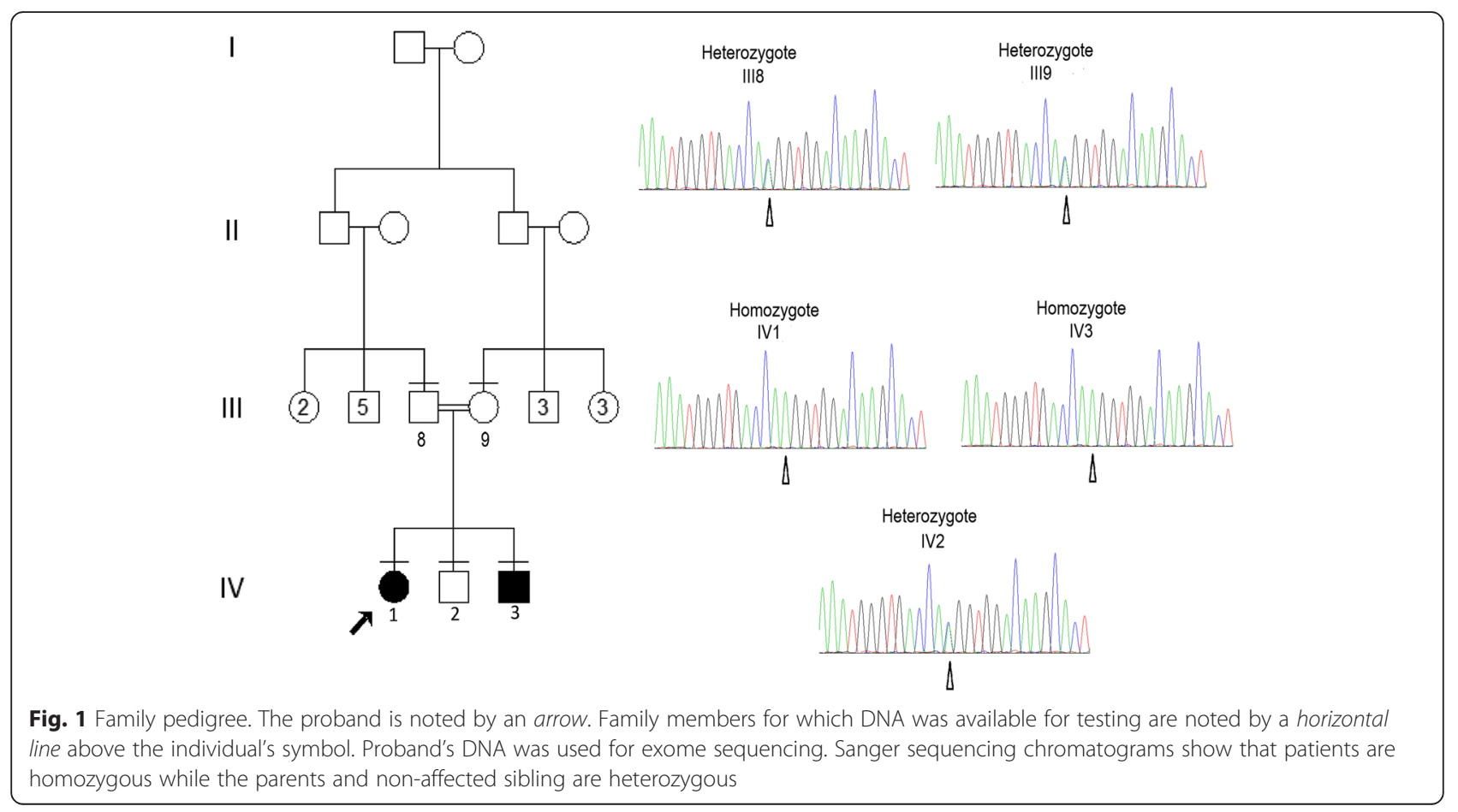


was $51 \mathrm{~cm}(-2 \mathrm{SD})$. Birth history was uncomplicated. She had hypotonia and motor delay; sat unsupported at 2 years and walked at 3 years. Speech development was also delayed. At 13 years of age, she shows hypotonia and intellectual disability $(\mathrm{IQ}=58$, Stanford-Binet scale). Her dysmorphic facial features (Fig. 2) include sparse eyebrows and eyelashes, pigmented sclerae, high forehead, prominent nose, low-set ears, and abnormal palmar creases. At 13 years, she showed no signs of puberty with axillary hair at A1, pubic hair at P1, breast development at B1, and absence of menarche. Investigations revealed a deficiency of growth hormone as measured by ITT and clonidinetesting. Radiologic examination revealed delayed bone age. Brain MRI, EEG, hearing and ophthalmologic testing were without abnormalities. Banded karyotype was 46, XX. The proband was reexamined at the age of 15 6/12 years showing a height of $135 \mathrm{~cm} \mathrm{(-3.8SD),} \mathrm{weight} \mathrm{of} 32 \mathrm{~kg}$ $(-2.3 \mathrm{SD})$, and $\mathrm{HC}$ of $51.2 \mathrm{~cm}(-2.2 \mathrm{SD})$. Menarche was reached at the age of 15 years.

The affected brother (IV:3) (Fig. 1) was first seen at the genetic clinic at the age of 5 years. He was delivered by cesarean section and noticed to have unilateral ptosis at birth. He had hypotonia; sitting unsupported at 2 years and walking at 3.5 years. Speech development was also delayed with first words at 2.5 years. Examination revealed a height of $85 \mathrm{~cm}$ (-4.8SD), weight of $13 \mathrm{~kg}$ $(-3 \mathrm{SD})$, and $\mathrm{HC}$ of $50 \mathrm{~cm}(-1.2 \mathrm{SD})$. He has intellectual disability with similar facies to his affected sister (Fig. 2) including sparse eyebrows and eyelashes, pigmented sclerae, unilateral ptosis, high forehead, prominent nose, long philtrum, low-set ears, and abnormal palmar creases. Investigations have shown similar results to his sister with growth hormone deficiency, delayed bone age, and no abnormalities in brain MRI, EEG, hearing, and ophthalmologic testing. Karyotype was 46, XY. He was reexamined at the age of 7 years showing a height of $94 \mathrm{~cm}(-4.8 \mathrm{SD})$, weight of $14 \mathrm{~kg}(-2.9 \mathrm{SD})$ and $\mathrm{HC}$ of $52 \mathrm{~cm}$ (within the mean) with intellectual disability $(\mathrm{IQ}=68$, Stanford-Binet scale).

\section{Genetic analysis}

In both parents, $56 \mathrm{ROH}$ were detected with a total size of 44 and $45.8 \mathrm{Mb}$ and average size of 785 and $817 \mathrm{~kb}$, respectively. In the children, the equivalent values were 58,65 , and $70 \mathrm{ROH}$, with a total size of 365,312 , and $114 \mathrm{Mb}$ and average size of $6.3,4.8$, and $1.6 \mathrm{Mb}$, respectively. The combination of the $\mathrm{ROH}$ provided the position of the target areas in which the candidate genes would be searched (Fig. 3).

After exome sequencing, 139,353,457 unique reads were on target, resulting in coverage of at least eight times for $98.1 \%$ of the protein coding fraction of the genome (RefSeq, version 58). Twenty-two thousend seven hundred sixty exonic variants of high quality were
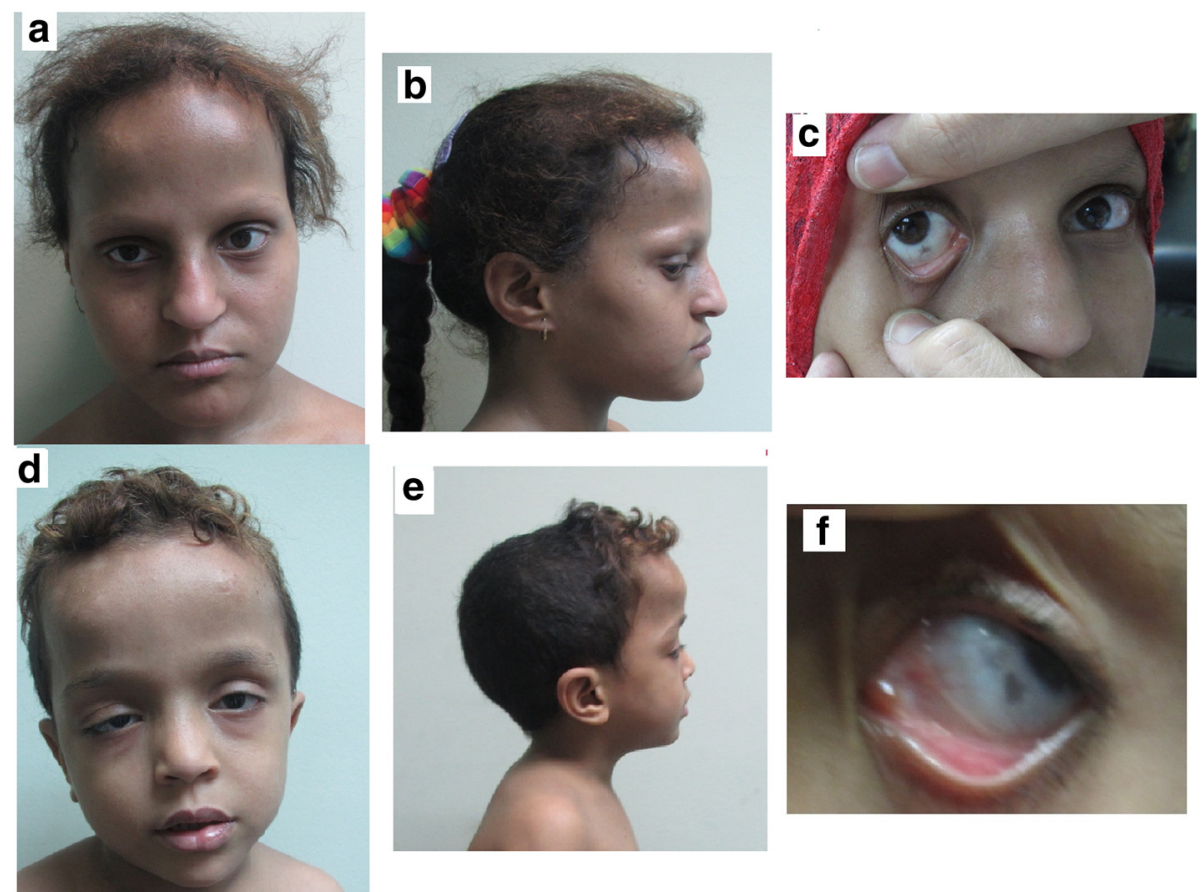

Fig. 2 Phenotypes of proband IV1 and brother IV3. a, b Proband's facial features with high forehead, prominent nose, and sparse eyebrows and eyelashes. c Scleral pigmentation in proband. d, e Facial features with high forehead, prominent nose sparse eyebrows and eyelashes, and unilateral ptosis in brother IV3. f Scleral pigmentation in brother IV3 


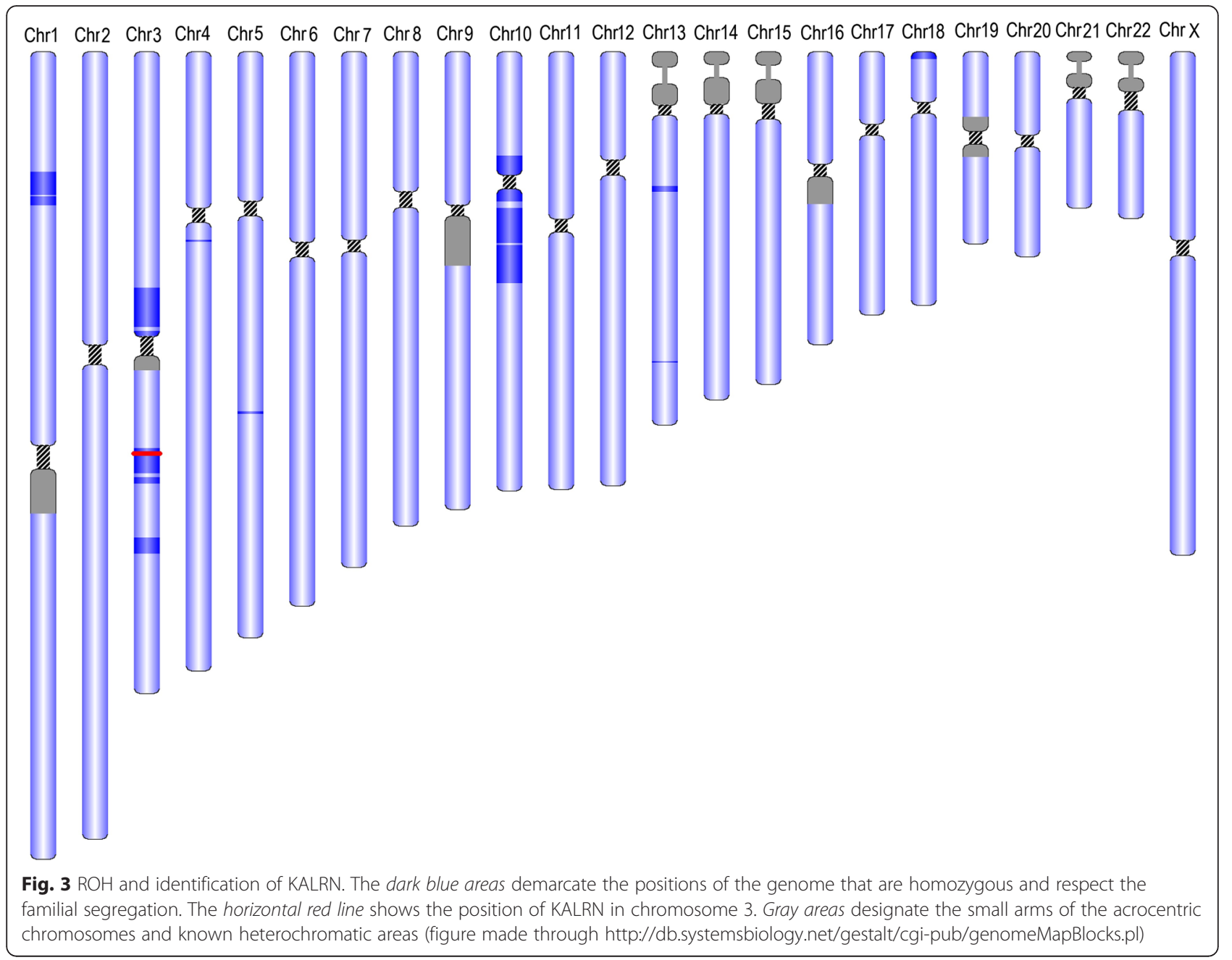

detected, 11,373 of which were synonymous (50.1\%), 10,044 (44.25\%) were missense, 74 (0.33\%) were nonsense, and 653 were indels $(2.87 \%)$.

Three variants passed the frequency criterion (MAF <0.01) and respected the segregation in the family. Of these, only one was predicted to be pathogenic by at least two pathogenicity prediction programs, NM_003947.4:c.3644C>A:p.(Thr1215Lys) in KALRN. The values predicted by SIFT [23], PolyPhen2 [24], and Mutation Taster [25] were 0.02, 0.8, and 0.996, respectively, and the region is very well conserved throughout the species (Fig. 4a). The variant was found in one of the previously defined target genomic areas of $6.6 \mathrm{Mbp}$ (Fig. 3). Sanger sequencing confirmed the homozygosity in the affected individuals and the heterozygosity of the identified variant in both parents and the nonaffected sibling. The variant is found in the ninth and last spectrin repeat of kalirin [26]. The spectrin repeats of kal7 are important for its role in spine morphogenesis [27, 28] (Fig. 4b).
The other two variants which were not predicted to be pathogenic were identified in CFAP57 (previously named WDR65) (NM_001195831.2:c.3299C>T:p.(Pro1100Leu)) and in the last exon in DPH2 (NM_001384.4:c.1429 C>T:p.(Arg477*)). CFAP57 was previously correlated with a Van der Woode syndrome (VDW) variant [29] but was later reclassified as variant of unknown clinical significance (VUS). Scarce data is known for DPH2 gene. In mouse, the function of the gene is mostly related to aging and to the skeleton, with expression in liver and biliary tissue. This data led us to consider this variant as unlikely to cause the phenotype in this family [30,31].

\section{Discussion}

Kalirin is a prime candidate for a role in ID since alterations in signaling pathways involving the Rho family of small GTPases, key regulators of the actin, and microtubule cytoskeletons contribute to both syndromic and non-syndromic intellectual disability disorders [32]. In mice, loss of one or both copies of Kalrn leads to 


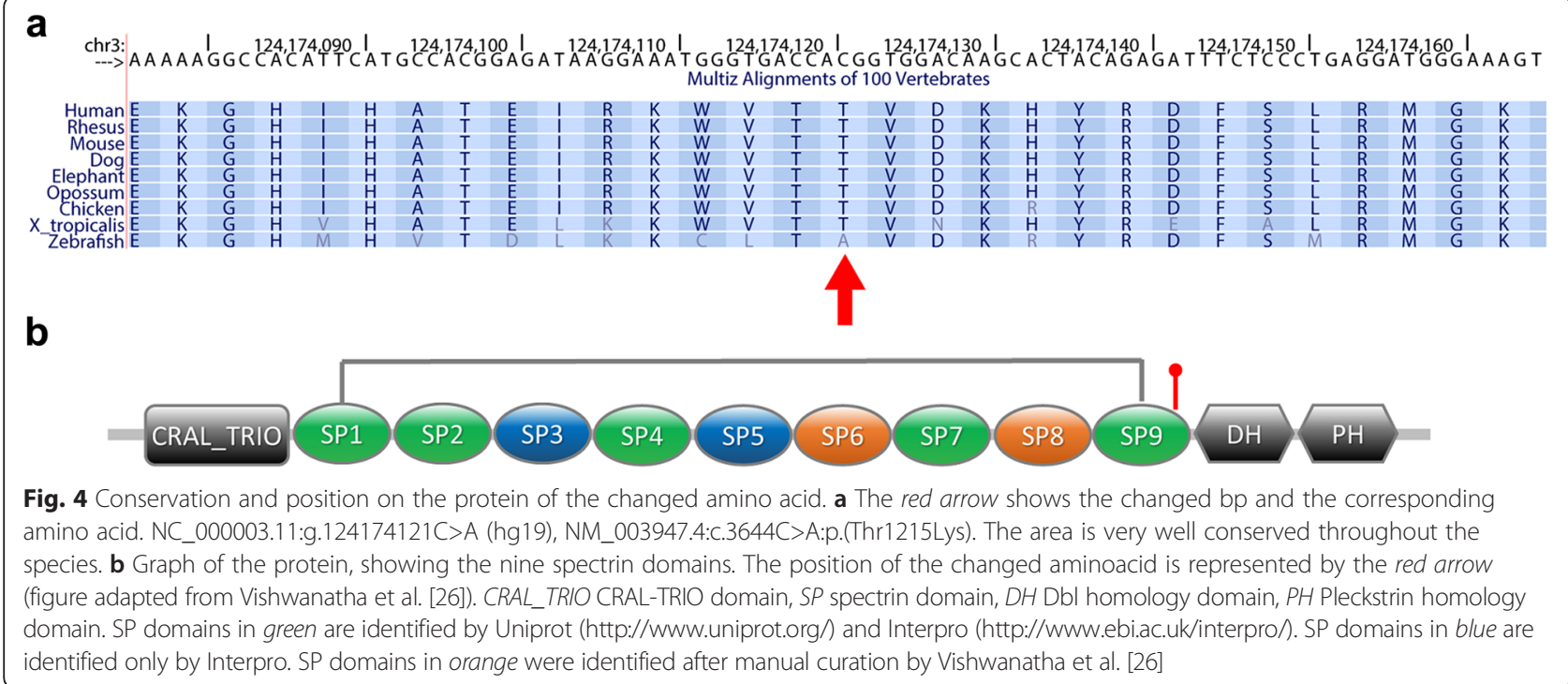

reduction in neuron spine densities in certain brain regions [33]. Heterozygote and knockout mice showed variable impairments in cognitive functions related to working memory, social recognition, and social approach, demonstrating the role of kalirin in the regulation of cortical ultrastructure and spine structural plasticity [34]. Elimination of Kalrn expression in POMC cells reduces anxiety-like behavior in mice [35]. Kalirin-7 in rodents (equivalent to human NM_003947.4) is the most abundant kalirin isoform in the adult rodent brain and is localized at the postsynaptic side of excitatory synapses [36, 37]. Knockout and overexpression studies revealed important roles for Kal7 in dendritic spine formation and synaptic function in rodents [16, 33, 34, 36].

The processes that regulate the morphological development of dendrites and dendritic spines have a significant impact on the establishment and function of synapses and on neuronal circuits [16]. The major functional group of ID-related proteins corresponds to proteins enriched at synaptic compartments [38], and investigations in children and adolescents with unclassified ID confirm the reduced density and spine dysgenesis in apical dendrites of the prefrontal cortex [39]. Abnormal neural cell spine morphology was the only anatomical alteration reported in cases of non-syndromic ID [33]. A rare coding variant, kalirin-7-D1338N, was identified in a schizophrenia patient and his sibling with major depressive disorder where both subjects carrying the polymorphism displayed reduced cortical volume in the superior temporal sulcus (STS), a region implicated in schizophrenia [40]. These data suggest that single amino acid changes in proteins involved in dendritic spine function can have significant effects on the structure and function of the cerebral cortex [40]. Other reports have indicated that dysfunctions in Rho-GEF signaling pathways are associated with various ID syndromes [41] and with Alzheimer disease [42]. Moreover, a correlation has been reported between the levels of Kalirin expression and the pathology of dendritic spines in some psychiatric and neurological disorders such as Huntington's disease, Alzheimer's disease, ischemic stroke, schizophrenia, depression, and cocaine addiction [43, 44].

In the tested family, there were clinical features other than ID, which could be correlated with Kalirin dysfunction. Both patients suffer from short stature and growth hormone deficiency. Mice with ablated spectrin domains of Kalirin had deficient growth rate and dysfunctional secretion of growth hormone [17]. Hypotonia was also present in our patients and may be related to the preand postsynaptic deficits in the neuromuscular junction reported in mice [17].

Kalirin has also been implicated in bone homeostasis where its deletion was reported to affect directly osteoclast and osteoblast activity. It may also play a role in paracrine and/or endocrine signaling events that control skeletal bone remodeling and the maintenance of bone mass [18]. This could be consistent with the observed delayed bone age in the patients.

The other two variants identified in CFAP57 and in the last exon in $D P H 2$ were not predicted to be pathogenic. The patients in the studied family do not have any of the features of VWS including the pits and/or sinuses of the lower lip, and cleft lip and/or cleft palate, which leads us to consider CFAP57 variant as a VUS in respect to the patient's phenotype. $D P H 2$, one of several enzymes involved in the synthesis of diphthamide linked to diphtheria toxin (OMIM 603456), was also considered as unlikely to cause the phenotype in this family. 


\section{Conclusions}

In this report, the clinical evaluation combined with the power and efficiency of genomic analysis defined a new candidate gene, $K A L R N$, as the possible underlying cause of the syndromic autosomal recessive ID in the studied family. The role of KALRN in causing cognitive impairment in human and the full phenotypic spectrum will be established when other families with ID show pathogenic variants in KALRN and the exact molecular pathophysiology is understood.

\section{Abbreviations \\ ID, intellectual disability; OMIM, Online Mendelian Inheritance in Man (http://omim.org/); ROH, run of homozygosity}

\section{Acknowledgements}

We are grateful to the members of the family enrolled in this study.

\section{Funding}

This study was supported by the grants from the Gebert ruf Stiftung foundation (to SEA), von Meissner foundation, and Bodossaki foundation (to PM).

\section{Availability of data and materials}

The variants will be deposited in the EGA EMBL-EBI.

\section{Authors' contributions}

$\mathrm{HH}, \mathrm{PM}$, and SEA designed the study and wrote the manuscript. PM performed the exome analyses. $\mathrm{HH}$ coordinated the patient collection. MZ and $\mathrm{Ml}$ examined the patients, described the phenotypic characteristics, and contributed the DNA samples. MG performed the exome sequencing. FAS conceived the used algorithms and MA coordinated Sanger sequencing. All authors contributed to the manuscript and approved the final version.

\section{Competing interests}

The authors declare that they have no competing interests.

\section{Consent for publication}

Parents of the affected children gave written consent for publication of photos.

\section{Ethics approval and consent to participate}

The study was approved by the Bioethics Committee of the University Hospitals of Geneva (protocol number: CER 11-036). All patients and/or parents provided their written informed consent for the analyses performed.

\section{Author details}

'Department of Genetic Medicine and Development, University of Geneva, 1 Rue Michel-Servet, 1211 Geneva, Switzerland. ${ }^{2}$ Service of Genetic Medicine, University Hospitals of Geneva, Geneva, Switzerland. ${ }^{3}$ Department of Clinical Genetics, National Research Centre, Cairo, Egypt. ${ }^{4}$ iGE3, Institute of Genetics and Genomics of Geneva, Geneva, Switzerland.

Received: 2 May 2016 Accepted: 5 July 2016

Published online: 16 July 2016

\section{References}

1. Al-Gazali L, Hamamy H, Al-Arrayad S. Genetic disorders in the Arab world. BMJ. 2006;333(7573):831-4.

2. Hamamy H, et al. Consanguineous marriages, pearls and perils: Geneva International Consanguinity Workshop Report. Genet Med. 2011;13(9):841-7.

3. Sheridan $\mathrm{E}$, et al. Risk factors for congenital anomaly in a multiethnic birth cohort: an analysis of the Born in Bradford study. Lancet. 2013;382(9901):1350-9.

4. Stoll C, et al. Parental consanguinity as a cause for increased incidence of births defects in a study of 238,942 consecutive births. Ann Genet. 1999;42(3):133-9.
5. Stoltenberg C, et al. Consanguinity and recurrence risk of birth defects: a population-based study. Am J Med Genet. 1999;82(5):423-8.

6. Makrythanasis $\mathrm{P}$, et al. Diagnostic exome sequencing to elucidate the genetic basis of likely recessive disorders in consanguineous families. Hum Mutat. 2014;35(10):1203-10.

7. Hamamy $\mathrm{H}$, et al. Recessive thrombocytopenia likely due to a homozygous pathogenic variant in the FYB gene: case report. BMC Med Genet. 2014;15:135.

8. Makrythanasis $\mathrm{P}$, et al. A novel homozygous mutation in FGFR3 causes tall stature, severe lateral tibial deviation, scoliosis, hearing impairment, camptodactyly, and arachnodactyly. Hum Mutat. 2014;35(8):959-63.

9. Makrythanasis $P$, et al. Pathogenic variants in PIGG cause intellectual disability with seizures and hypotonia. Am J Hum Genet. 2016;98(4):615-26.

10. Musante L, Ropers HH. Genetics of recessive cognitive disorders. Trends Genet. 2014;30(1):32-9.

11. Vissers LE, Gilissen C, Veltman JA. Genetic studies in intellectual disability and related disorders. Nat Rev Genet. 2016;17(1):9-18.

12. Najmabadi $\mathrm{H}$, et al. Deep sequencing reveals 50 novel genes for recessive cognitive disorders. Nature. 2011;478(7367):57-63.

13. Anazi $\mathrm{S}$, et al. A null mutation in TNIK defines a novel locus for intellectual disability. Hum Genet. 2016;135(7):773-8.

14. Iqbal Z, et al. Homozygous SLC6A17 mutations cause autosomal-recessive intellectual disability with progressive tremor, speech impairment, and behavioral problems. Am J Hum Genet. 2015;96(3):386-96.

15. Philips AK, et al. Identification of C12orf4 as a gene for autosomal recessive intellectual disability. Clin Genet. 2016. Jun 17. doi:10.1111/cge.12821. [Epub ahead of print]

16. Cahill ME, et al. Kalirin regulates cortical spine morphogenesis and disease-related behavioral phenotypes. Proc Natl Acad Sci U S A. 2009;106(31):13058-63.

17. Mandela P, et al. Kalrn plays key roles within and outside of the nervous system. BMC Neurosci. 2012;13:136.

18. Huang $\mathrm{S}$, et al. The Rho-GEF kalirin regulates bone mass and the function of osteoblasts and osteoclasts. Bone. 2014;60:235-45.

19. Li H, et al. The sequence alignment/map format and SAMtools. Bioinformatics. 2009;25(16):2078-9.

20. Ye K, et al. Pindel: a pattern growth approach to detect break points of large deletions and medium sized insertions from paired-end short reads. Bioinformatics. 2009:25(21):2865-71.

21. Wang K, Li M, Hakonarson H. ANNOVAR: functional annotation of genetic variants from high-throughput sequencing data. Nucleic Acids Res. 2010;38(16), e164

22. Santoni FA, Makrythanasis P, Antonarakis SE. CATCHing putative causative variants in consanguineous families. BMC Bioinformatics. 2015;16(1):310.

23. Ng PC, Henikoff S. Predicting deleterious amino acid substitutions. Genome Res. 2001;11(5):863-74.

24. Adzhubei IA, et al. A method and server for predicting damaging missense mutations. Nat Methods. 2010;7(4):248-9.

25. Schwarz JM, et al. MutationTaster2: mutation prediction for the deep-sequencing age. Nat Methods. 2014;11(4):361-2.

26. Vishwanatha KS, et al. Structural organization of the nine spectrin repeats of kalirin. Biochemistry. 2012;51(28):5663-73.

27. Schiller MR, et al. Autonomous functions for the Sec14p/spectrin-repeat region of kalirin. Exp Cell Res. 2008;314(14):2674-91.

28. Ma XM, et al. Nonenzymatic domains of kalirin7 contribute to spine morphogenesis through interactions with phosphoinositides and Abl. Mol Biol Cell. 2014;25(9):1458-71.

29. Rorick NK, et al. Genomic strategy identifies a missense mutation in WDrepeat domain 65 (WDR65) in an individual with Van der Woude syndrome. Am J Med Genet A. 2011;155A(6):1314-21.

30. Eppig JT, et al. The Mouse Genome Database (MGD): facilitating mouse as a model for human biology and disease. Nucleic Acids Res. 2015;43(Database issue):D726-36.

31. Smith CM, et al. The mouse Gene Expression Database (GXD): 2014 update. Nucleic Acids Res. 2014;42(Database issue):D818-24.

32. Newey SE, et al. Rho GTPases, dendritic structure, and mental retardation. J Neurobiol. 2005;64(1):58-74.

33. Vanleeuwen JE, Penzes P. Long-term perturbation of spine plasticity results in distinct impairments of cognitive function. J Neurochem. 2012;123(5):781-9.

34. Xie Z, Cahill ME, Penzes P. Kalirin loss results in cortical morphological alterations. Mol Cell Neurosci. 2010;43(1):81-9. 
35. Mandela $\mathrm{P}$, et al. Elimination of Kalrn expression in POMC cells reduces anxietylike behavior and contextual fear learning. Horm Behav. 2014;66(2):430-8.

36. Ma XM, et al. Kalirin-7, an important component of excitatory synapses, is regulated by estradiol in hippocampal neurons. Hippocampus. 2011;21(6):661-77.

37. Miller MB, et al. Neuronal Rho GEFs in synaptic physiology and behavior. Neuroscientist. 2013;19(3):255-73.

38. Pavlowsky A, Chelly J, Billuart P. Emerging major synaptic signaling pathways involved in intellectual disability. Mol Psychiatry. 2012;17(7):682-93.

39. Kaufmann WE, Moser HW. Dendritic anomalies in disorders associated with mental retardation. Cereb Cortex. 2000;10(10):981-91.

40. Russell TA, et al. A sequence variant in human KALRN impairs protein function and coincides with reduced cortical thickness. Nat Commun. 2014;5:4858.

41. Ramakers GJ. Rho proteins, mental retardation and the cellular basis of cognition. Trends Neurosci. 2002;25(4):191-9.

42. Youn $\mathrm{H}$, et al. Kalirin is under-expressed in Alzheimer's disease hippocampus. J Alzheimers Dis. 2007;11(3):385-97.

43. Mandela P, Ma XM. Kalirin, a key player in synapse formation, is implicated in human diseases. Neural Plast. 2012;2012:728161.

44. Remmers C, Sweet RA, Penzes P. Abnormal kalirin signaling in neuropsychiatric disorders. Brain Res Bull. 2014;103:29-38.

Submit your next manuscript to BioMed Central and we will help you at every step:

- We accept pre-submission inquiries

- Our selector tool helps you to find the most relevant journal

- We provide round the clock customer support

- Convenient online submission

- Thorough peer review

- Inclusion in PubMed and all major indexing services

- Maximum visibility for your research

Submit your manuscript at www.biomedcentral.com/submit
) Biomed Central 